\title{
Colias IV: the Affordable Micro Robot Platform with Bio-inspired Vision
}

\author{
Cheng $\mathrm{Hu}^{1[0000-0002-1177-2167]}$, Qinbing $\mathrm{Fu}^{1[0000-0002-5726-6956]}$, and \\ Shigang Yue ${ }^{1[0000-0002-1899-6307]}$ \\ Lincoln Centre for Autonomous Systems Research, University of Lincoln, UK, \\ $\{$ chu, qifu, syue\}@lincoln.ac.uk
}

\begin{abstract}
Vision is one of the most important sensing modalities for robots and has been realized on mostly large platforms. However for micro robots which are commonly utilized in swarm robotic studies, the visual ability is seldom applied or with reduced functions/resolution, due to the high demanding on the computation power. This research has proposed the low-cost micro ground robot Colias IV, which is particularly designed to meet the requirements to allow embedded vision based tasks on-board, such as bio-inspired collision detection neural networks. Numerous of successful approaches have demonstrated that the proposed micro robot Colias IV to be a feasible platform for introducing visual based algorithms into swarm robotics.
\end{abstract}

Keywords: micro robot, bio-inspired, vision, collision detection, lowcost

\section{Introduction}

Vision is one of the most important sensing modalities for autonomous robots since it provides the abundant and reliable information about the surrounding environment. Thanks to the development in computer vision technology, many large-scale robots are taking advantage from vision sensors and also the most advanced vision algorithms [1].

On the other hand, small-scale robots, especially micro robots that are usually employed in the swarm robotic research, can hardly benefit from visual sensing. Even though the growth of interest in swarm robotics has brought us a wide range to choose from [2-6], there is still no optimal design for all desires. One of the greatest challenges is the trade-off between limited computational resources on-board and the requirements for image processing in real-time. The lack of vision inputs for swarm robotics has become a major concern for researchers recently. For example, the E-puck [2], Swarm-bot [3] or mROBerTO [4] are short of RAM size or sufficient CPU frequency. On the other hand, for those robots whose CPU are strong enough, they are usually oversized for swarm scenarios, such as Kobot [5], Wolfbot [6] and Khepera III. The comparison of related robot platform are illustrated in table 1. 
Table 1: The Compare of Cutting-edge Robot Platforms with Image Sensors

\begin{tabular}{ccccccc}
\hline & Kobot & \multicolumn{1}{c}{ KheperaIII E-puck } & Swarm-Bot & mROBerTO & Colias IV \\
\hline CPU(MHz) & 200 & 600 & 64 & 40 & 16 & 180 \\
RAM & $32 \mathrm{M}$ & $32 \mathrm{M}$ & $8 \mathrm{~K}$ & $648 \mathrm{~K}$ & $32 \mathrm{~K}$ & $256 \mathrm{~K}$ \\
cost $(£)$ & 800 & 2000 & 580 & 500 & $50^{*}$ & $80^{*}$ \\
diameter(cm) & 12 & 7 & 7.5 & 12.7 & 2.2 & 4 \\
sensor types & 4 & 7 & 4 & 4 & 7 & 6 \\
autonomy(h) & 10 & 1 & 1 & 3 & $1.5-6$ & 1.5 \\
remarks & Undeveloped & discontinue & & & *parts & *parts \\
\hline
\end{tabular}

For micro robots, suitable vision based algorithms should be selected and applied $[2,7]$. For example, the bio-inspired collision detection model Lobular Giant Movement Detector (LGMD) inspired from locust visual neurons [8,9]. Recently, the LGMD neuron has been modelled into computational algorithms for autonomous vehicles [10-12]. However, due to the requirements on hardware resources for image processing, the available robotic platforms or workaround approaches are unsatisfactory: either too large [10], too expensive [11], or can't get rid of the connecting with a host device (wire or wirelessly) to process the vision model [12]. These inconveniences prevent this promising visual model to be applied in swarm robotic researches for further study. An affordable micro robot platform that is small enough and can work independently is necessary.

In this paper, we propose a novel design of micro robot Colias $I V$ to satisfy mentioned requirements, which is an affordable hardware platform mainly deals with visual tasks. As an upgrade version of previously develop micro robot Colias [13] that is a differential driven ground robot with modular design, the circular footprint occupies only $40 \mathrm{~mm}$ in diameter. The new Colias $I V$ is additionally featured with an strong ARM Cortex M4 processor, a tiny VGA camera, two digital microphones, one 9-axis motion sensors and various of other sensing modules. To develop algorithms on the platform easily, software packages are provided, including the motion planning and controlling strategies, a friendly embedded programming environment and remote user accessibility through extension modules. The bio-inspired collision detection algorithm called Embedded-LGMD(ELGMD) and other related visual neural networks have been successfully realized on this micro robot, which shows its practicability on conducting vision based tasks autonomously.

\section{The Assembly of Colias IV}

The designed micro robot platform is based on three primary objectives: 1) to realize middle-strength computation tasks including low-level image processing autonomously, 2) to achieve better modularity so that each part has certain features and functions that can work independently, and 3) to maximize the use of space to save as much space occupation as possible.

As a result of the objectives, the robot is composed of three modularized layers that provide different functions, namely the Colias Basic Unit (CBU), 


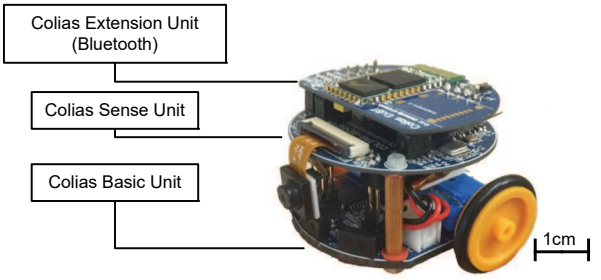

(a)

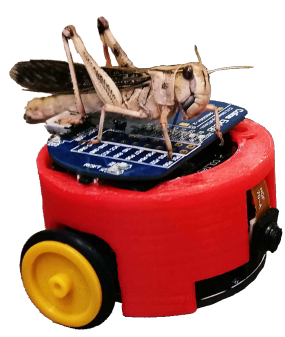

(b)

Fig. 1: (a)The Colias IV robot with CEU (Bluetooth) board attached, showing the modules' connections. The battery is located between the CSU and CBU; (b) A Colias $I V$ coated with a 3D-printed shell and CEU (USB-serial). An adult locust is standing on the robot to compare the size.

the Colias Sensing Unit (CSU) and the Colias Extension Units (CEU). The $\mathrm{CBU}$ serves preliminary robot features such as motion, power management and some basic sensing. The CSU provides the high-level sensors such as motion sensors and camera towards better programming environment with image processing. Robot formed by $\mathrm{CBU}$ and CSU is sufficient for most of the experiments. Furthermore, CEU provides specialized features such as connectivity and illumination. The overall weight of Colias IV is $40-60 \mathrm{~g}$, varied according to different extensions. The hardware architecture of Colias IV is illustrated in figure 2 .

\subsection{The Colias basic unit (CBU)}

The Colias basic unit (CBU) is a upgraded version of previous Colias robot started in 2013 [13], which is a light weight robot designed for swarm applications. It is one of the smallest and cheapest micro-robots in this field. CBU employs a circular platform with a diameter of $40 \mathrm{~mm}$. It serves basic sensing, motion generation and power management. The schematic design of the CBU is illustrated in figure 2, and a photograph of CBU is shown in figure 3.

Micro Processor The purpose of CBU is basic motion control and sensing, thus the primary consideration for choosing the processor is more of stability and power-efficiency but not computation speed. The AVR series micro-controller ATMega168P from Atmel is selected to meet these requirements. It is an 8-bit micro-controller running at $8 \mathrm{MHz}$, equipped with 16 KBytes Flash, 1 KByte RAM. It has 24 IO pins supporting analog-digital converter and serial ports.

The application program inside the CBU's is arranged with an infinite loop. In the determined duty cycle of approximately $2 \mathrm{~ms}$, the micro controller manages to update a specific area of RAM as a register map. The register map maintains the CBU's system status including sensor data, LED configuration, 


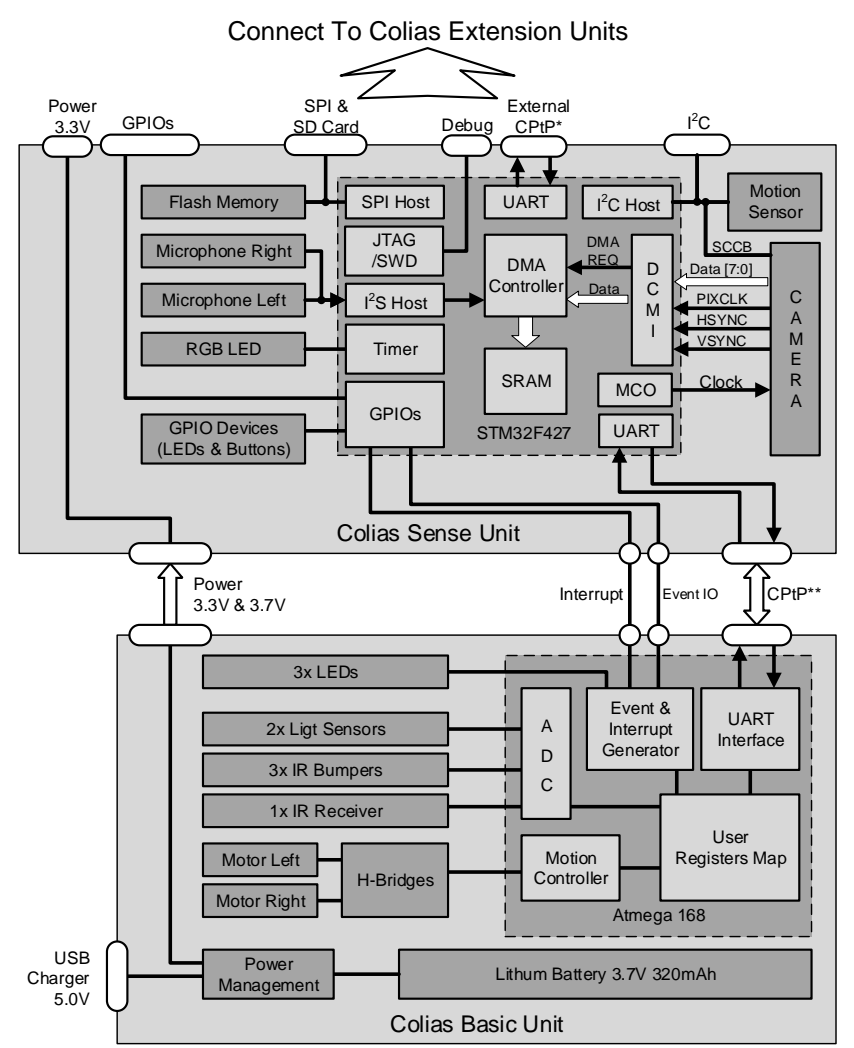

Fig. 2: The Colias IV block diagram, showing the CBU and CSU architecture. ${ }^{*} \mathrm{CPtP}$ is a reliable data transmission protocol that utilizing the UART as the physical layer.

motion configuration and also system information. There is a separate interrupt handle to decode and response to incoming control messages from CSU or other higher level modules.

Environment Sensors The CBU is equipped with three kinds of sensors, which are short range infra-red (IR) proximity sensors facing front, ambient light sensors facing ground, and one long range IR receiver towards back.

The short range IR sensors (bumpers) are commonly used to indicate real collisions. Each bumper has a IR emitter and a receiver towards the same direction. Confirmation of collision is relied on the strength of received signal. There are three bumper sensors facing front separated by $30^{\circ}$. Their average detection distance is $20 \mathrm{~mm}$, which is also influenced by the light absorbing character of the obstacle (figure 4a). The ambient light sensors are responsible to measure light intensity within the spectrum of visible light with a phototransistor. They are mounted towards the ground on both sides of the robot, 


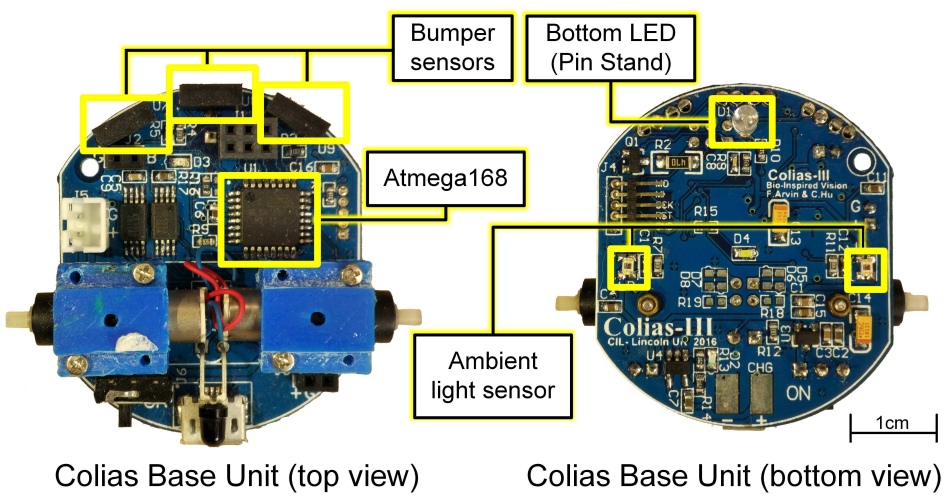

Fig. 3: The photo of CBU from both sides with wheels and battery removed, indicating several major components. In the bottom view, notice that the bottom LED that also acts as the front pin stand.

which enable the robot to follow patterns on the ground, or react to the surrounding illumination status. There is a long range IR receiver mounted to the back, which is applied to receive signals from IR remote controller and trigger actions accordingly. This feature improves the efficiency when conducting experiments on multiple robot agents.

Locomotion The Colias IV employs differential driving method with two mini geared DC motors. Each motor is driven through a H-bridge using pulse width modulation (PWM) for power tuning. To support the robot with only two wheels, the front LED is used as auxiliary fixed point stand, as indicated in figure 3. The tested performance of speed tuning is shown in figure $4 \mathrm{~b}$ with battery conditions of full charged and normal levels. Due to the compact design that prohibits encoders to be mounted on-board, the rotation speed of wheels can not be measured directly. However the attitude of the robot can be estimated through motion sensors described in section 2.2.

Contributed by the high gear ratio (136:1) and lightweight of the robot load $(50 \mathrm{~g})$, the acceleration process could be achieved in several milliseconds. The dynamics of the motor is normally not taken into consideration when modelling the motion. The kinematics of the robot motion is described as a simple differential driven model. Let $v_{l}$ and $v_{r}$ donate to the left and right wheel velocities, we got the equation to represent the desired motion at any moment:

$$
\begin{aligned}
& \omega\left(R+\frac{l}{2}\right)=v_{l} \\
& \omega\left(R-\frac{l}{2}\right)=v_{r}
\end{aligned}
$$

where $\omega$ is the angular velocity of the robot body, $l$ is the distance between the wheels and $R$ donates the distance between the midpoint of the wheels and Instant Centre of Rotation $(I C R)$. 


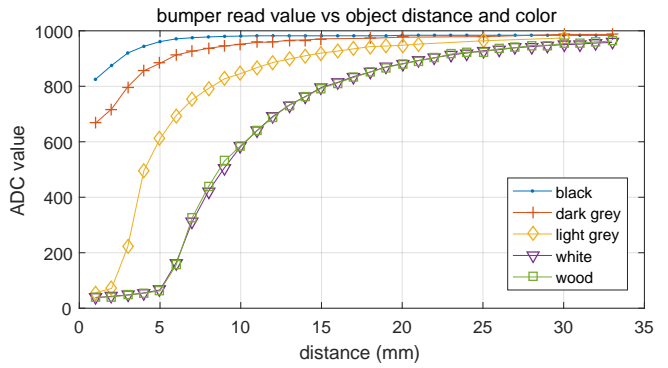

(a)

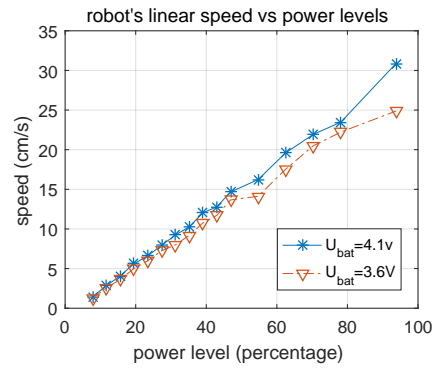

(b)

Fig. 4: Tests on some of the CBU features. (a) The short range bumper read-out value against object distance with different colour. The white object: normal white paper; the light grey to black: printing with $25 \%, 75 \%$ and $100 \%$ densities; wood: pine wood surface; (b) The robot's linear moving speed (forward motion) vs PWM controlled power level in battery conditions: full battery $(4.1 \mathrm{~V})$ and nominal battery level(3.6V).

The robot can be operated with open-loop movement control when precise motion is not required. It can also achieve close-loop motion control by buildin PID controllers when the motion sensor (gyroscope) is used to estimate the attitude of the robot itself. The PID controllers are available for either turning speed or the heading angle servo, as well as their combination that forms a cascade controller to increase the overall dynamic response.

Power Management The robot is powered by a Li-Ion battery with the capacity of $320 \mathrm{mAh}$ (milliamp Hours) which provides an autonomy of approximately 1.5 hours for the robot. The nominal voltage is $3.7 \mathrm{~V}$. The battery charging process, which is managed by a charging monitor chip TP4054, can be supplied through either an USB-micro port or a pair of charging shoes at the bottom of the board at $5 \mathrm{~V}$. The whole robot is powered by this battery through a linear regulator at $3.3 \mathrm{~V}$ except the motors, which are directly powered by the battery. The power consumption of typical components are listed in table 2 . The power consumption of the robot under normal conditions (in a basic arena with only walls) and short-range communication (low-power IR emitters) is about $150 \mathrm{~mW}$. However, it can be reduced to approximately $30 \mathrm{~mW}$ when IR emitters are turned off occasionally.

\subsection{The Colias sense unit (CSU)}

The Colias Sense Unit (CSU) is the most important layer in the platform. It processes the main sensing task and most of the computation tasks. The earlier version of CSU was described as the extension vision module attaching to the formal Colias robot in previous research $[14,15]$, which has only the camera sensor installed. The redesigned CSU now provides variety of sensors including 
Table 2: The Power Consumptions of Major Components

\begin{tabular}{clll}
\hline Module Name \& Description & typical & max & unit \\
\hline Processor I active & 2.5 & 8 & \\
Processor II standby ** & 2.5 & & \\
Processor II active & 95 & 130 & \\
Camera active & 12 & 18 & $m A$ \\
IR Sensors x3 & 9 & 45 & \\
Motion Sensor & 2 & 4.7 & \\
DC Motor x2 & $80(10 \mathrm{~cm} / \mathrm{s})$ & $300(30 \mathrm{~cm} / \mathrm{s})$ & \\
\hline
\end{tabular}

* This is the AVR chip in Colias Basic Unit.

** This is the STM32F427 chip in Colias Sensing Unit.

the CMOS camera, two Microelectromechanical systems (MEMS) microphones with digital outputs and a 9-DOF (domain of freedom) motion sensor. All the sensors are connected to the powerful ARM processor. The CSU is also equipped with an external Flash chip with up to 256 Mbits for permanent data storage. With external sockets for multiple interfaces, extension modules can be mounted to the CSU, providing features such as inter-robot long range communication, illumination or even physical grippers. The photograph of CSU is shown in figure 5 , and the schematic of CSU is shown in figure 2. The CSU is connected to the CBU with three group of pin headers for power supply and communication. A reliable communication protocol is designed and applied here for exchanging message between the two units.

Main Processor An ARM Cortex-M4F core micro controller is deployed as the main processor in the CSU that can handle intensive image processing and monitoring all other modules including the camera, CBU and other sensors. The 32-bit Micro Control Unit (MCU) STM32F427 is an upgraded version of which previously deployed in $[14,15]$ (STM32F407). Several features make the STM32F 427 chip an ideal platform for the designed objectives. For example, the high-performance processor with Reduced Instruction Set Computer(RISC) technology and the floating processing units (FPU) running at a high speed of $180 \mathrm{MHz}$ donate for effectiveness and low power consumption; the 256 KByte SRAM and 2 MByte Flash provide the necessary storage space for image buffering; the on-board peripheral digital camera interface (DCMI), which is a high speed data interface for receiving camera data, frees the CPU from the hard burdens of managing large amount of data transferring. Moreover, the package size of STM32F427 is only $14 \mathrm{~mm} \times 14 \mathrm{~mm}$ with quad-flat-package (QFP) footprint. Comparing to stronger chips that is often packed as Ball Grid Array (BGA), QFP is more favourable in two-layer PCB layout design, which is cost-efficient for manufacturing and testing.

The major part of implemented algorithms are realized in embedded $\mathrm{C} / \mathrm{C}++$. Since there are lots of tasks to be managed asynchronously and periodically, 
the recommended software architecture is the "interrupt-controlled loop" to ensure minimum latency and memory occupation on tasks dispatching. To provide a friendly programming environment for developers so that background tasks are isolated from the user codes, a supporting package is designed to manipulate peripheral devices and communication interfaces as a brunch of handles, including the camera driver, the motion sensory data calculation, I/O devices and the CBU commands. The organization of this package is compatible and inheritable with the hardware abstract layer (HAL) drivers package provided by STMicroelectronics.

Camera A low voltage CMOS image sensor OV7670 module is utilised in CSU, as it is a low-cost camera with a compact package size of $8 \times 4 \mathrm{~mm}^{\mathbf{3}}$ with flexible flat fable (FFC) connector. The power supply is $3.3 \mathrm{~V}$ with active power consumption of up to $60 \mathrm{~mW}$. The camera is capable of operating up to 30 frames per seconds (fps) in VGA mode with output support for various colour formats. The horizontal viewing angle is approximately $70^{\circ}$. As a trade-off for image quality and memory space, the resolution is configured at $72 \times 99$ pixel on $30 \mathrm{fps}$, with output format of 8-bit YUV422. This image format separates each pixel's colour channels from the brightness channel, thus no additional colour transforming operations are required when only brightness information is required in further processing. The camera is connected to the MCU with two groups of interfaces, which are a serial camera control bus (SCCB) for camera configuration, and a group of image data/synchronization signals through DCMI interface, as shown in figure 2 .

Other Sensor One 9-DoF (degrees of freedom) motion sensor MPU9250 which contains a gyroscope, an accelerometer and a magnetometer is installed in the CSU which enables Colias to detect its attitude. The motion sensor is connected to the $\mathrm{MCU}$ via $\mathrm{I}^{2} \mathrm{C}$ interface with the refresh rate of $20 \mathrm{~Hz}$. The robot's orientation is periodically estimated by a differential equation:

$$
\left[\begin{array}{c}
\dot{\gamma}_{t+1} \\
\dot{\theta}_{t+1} \\
\dot{\psi}_{t+1}
\end{array}\right]=\frac{1}{\cos \theta_{t}}\left[\begin{array}{ccc}
\cos \theta_{t} & \sin \gamma_{t} \sin \theta_{t} & \cos \gamma_{t} \sin \theta_{t} \\
0 & \cos \theta_{t} \cos \gamma_{t} & -\sin \gamma_{t} \cos \theta_{t} \\
0 & \sin \gamma_{t} & \cos \gamma_{t} \cos \theta_{t}
\end{array}\right]^{-1} \cdot\left[\begin{array}{l}
\omega_{t}^{E b X} \\
\omega_{t}^{E b Y} \\
\omega_{t}^{E b Z}
\end{array}\right]
$$

where the left part is refreshed robot's orientation, represented by Euler angles (roll, pitch and yaw) and the right part is the estimated orientation last time. $\omega_{t}^{E b X}, \omega_{t}^{E b Y}$ and $\omega_{t}^{E b Z}$ are acquired rotation speed around three axes from the IMU sensor. In this attitude estimation approach, there is a zero point shifting problem caused by the nature of gyroscope, leading to an increasing accumulated error. According to our tests, this error is less than $2^{\circ}$ per minute, yet acceptable enough for simple behaviour generation in our experiments.

Two MEMS microphones are also applied in the CSU. Each microphone has the frequency response of $60-15 \mathrm{kHz}$ with sensitivity up tp $-26 \mathrm{dBFS}$. The omnidirectional microphones are placed with a distance of $33 \mathrm{~mm}$, connected 


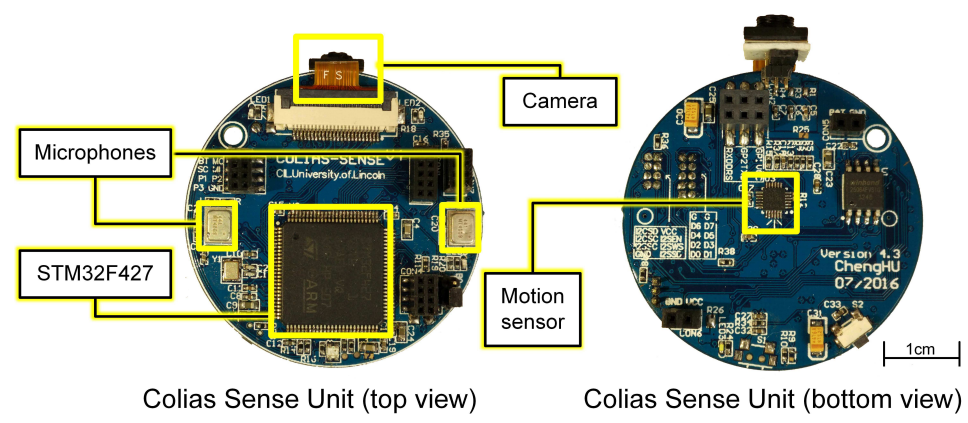

Fig. 5: The photo of CSU from both sides. Showing some major components.

with the main processor through $\mathrm{I}^{2} \mathrm{~S}$ interface. The power consumption is $1.4 \mathrm{~mA}$ each. The microphones are reserved for future applications. For example, by analysing the phase delay between the pair of microphones from a single sound source, the source localization and tracking tasks could be achieved.

\subsection{The Colias Extension Units (CEU)}

We have inserted as many peripherals into the tiny boards of CBU and CSU, enabling Colias $I V$ to perform most of the experiments independently. Yet some further features which consume large space but not required by all experiments, or only required for debugging sessions such as USB interface, Bluetooth data transmitter, wireless first person view transmitter [16] or other local communication oriented sensors, are achieved by removable extension units connected to CSU through the extension sockets.

Currently we have realized four different types of CEUs. The USB module and Bluetooth module are used in the research mostly. The USB extension module serves the main purpose of debugging, downloading binary program into the CSU, and transmit massive data like a frame of image through an USB cable. The maximum bandwidth is $3 \mathrm{MBit} / \mathrm{s}$. The Bluetooth extension module enables the Colias IV to communicate with a remote host device such as a laptop or a smart-phone, receiving motion commands or sending sensor data. The maximum bandwidth is $512 \mathrm{KBit} / \mathrm{s}$. Both USB and Bluetooth extension modules are equipped with an SD card slot and eight LEDs, which are extremely useful in experiments with multiple robots that temporary data storage is needed.

\section{Bio-inspired visual motion sensing: case studies}

Benefiting from the friendly developing environment, several successful bioinspired visual motion sensing model such as the LGMD1 [16,17], LGMD2 [15] and DSN [18] could be realized inside the Colias IV robot, enabling them to work independently to recognize fast approaching objects and trigger maneuver commands such as collision avoidance. 
10

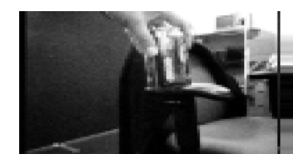

(a)

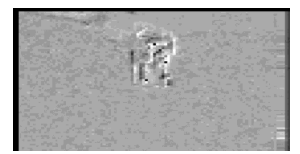

(b)

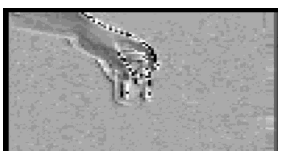

(c)

Fig. 6: The sample of LGMD1 layers during a single process. (a) shows the original input image, that a can of beer is waving in front of the robot; (b) shows the result of summing layer (the sum of the excitation layer and the inhibition layer) that filters out stationary background; (c) shows the grouping layer, which enhancing the filtered moving foregrounds. (figure adapted from [17])
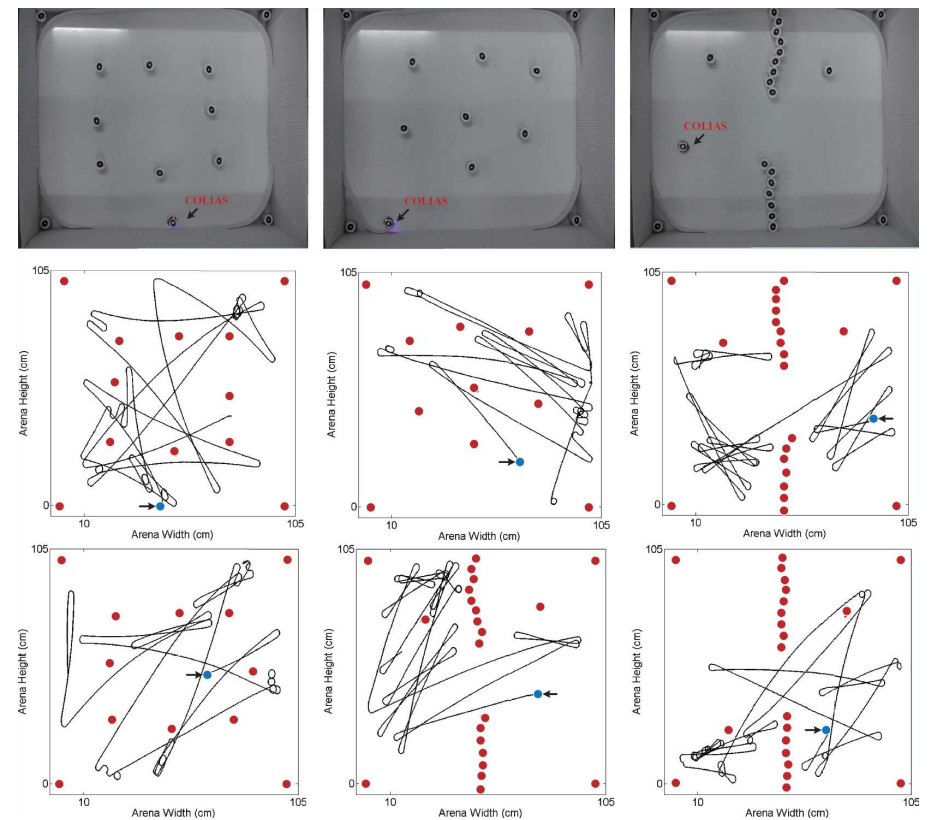

Fig. 7: The experiment of a Colias $I V$ robot with embedded LGMD2 model to detect imminent collisions. The robot is allowed to wander inside the arena with distributed obstacles. The red dots indicate the obstacles. (figure adapted from [15])

The embedded LGMD1 model (also called the ELGMD) is a layered neural model formed by five layers with lateral inhibition mechanism and two single cells [17]. The computational model contains only low-level image processing such as excitation transferring and neighbouring operations. With latest optimizations for the embedded processor, it can achieve CPU time occupation of 7-10 ms, and the RAM occupation of around 38 KBytes. In previous experiments with embedded LGMD1, the robot is able to run autonomously inside an square arena full of obstacles for more than 10 min without collisions. Details of the structure of ELGMD, its realization on Colias IV and the experiments are available in [17].

In locust, the LGMD2 is a neighbouring neural model identified next to the LGMD1. They share lots of similarities on structure and neural responses 
towards approaching objects. The difference is that the LGMD2 does little or no response to bright approaching objects in front of dark background [19]. This special mechanism which enhance the model's foreground selectivity is also realized in the embedded LGMD2 model with the Colias IV robot [15]. Their different responses towards visual looms are also demonstrated and analysed in arena experiments with several robots equipped with both the LGMD1 and LGMD2 model, but their input visions are two overlapping parts from a single frame [16]. The example of these experiments are represented in figure 7.

All these embedded visual models are developed on the Colias IV robot platform, which demonstrates its feasibility and further potential for researching bio-inspired visual models, or other computational demanding models on-board when multi sensory data is required.

\section{Summary}

A miniature ground mobile robot Colias IV occupying footprint of $4 \mathrm{~cm}$ in diameter is developed to study computational intensive embedded models. Featured by a strong ARM processor, variety of sensors including a tiny camera and two digital microphones, and enormous capabilities of connectivity, this micro robot can realize bio-inspired visual detecting model such as LGMD1 and other related neural models on-board in real time, which have been tested and studied by a series of experiments.

Even though with compact size and low cost, the developed robot Colias $I V$ has shown potential for further research based on multi-agent experiments such as the aggregation behaviours in robot swarms. But challenges still exist, for example, to coordinate a group of robots spontaneously, the required communication among each other is difficult to be accomplished without global synchronization. This could be solved by utilizing an extension board with additional communication modules, relying on either RF or optical approaches.

Acknowledgement This work was supported by the EU FP7 project HAZCEPT(318907) and Horizon 2020 project STEP2DYNA (691154).

\section{References}

1. I. Kostavelis and A. Gasteratos, "Semantic mapping for mobile robotics tasks: A survey," Robotics and Autonomous Systems, vol. 66, pp. 86-103, 2015.

2. J. Chen, M. Gauci, W. Li, A. Kolling, and R. Groß, "Occlusion-based cooperative transport with a swarm of miniature mobile robots," IEEE Transactions on Robotics, vol. 31, no. 2, pp. 307-321, 2015.

3. M. Dorigo, "Swarm-bot: An experiment in swarm robotics," in Swarm Intelligence Symposium, 2005. SIS 2005. Proceedings 2005 IEEE. IEEE, 2005, pp. 192-200.

4. J. Y. Kim, T. Colaco, Z. Kashino, G. Nejat, and B. Benhabib, "mroberto: A modular millirobot for swarm-behavior studies," in Intelligent Robots and Systems (IROS), 2016 IEEE/RSJ International Conference on. IEEE, 2016, pp. 21092114. 
5. A. E. Turgut, F. Gokce, H. Celikkanat, L. Bayindir, and E. Sahin, "Kobot: A mobile robot designed specifically for swarm robotics research," Middle East Technical University, Ankara, Turkey, METUCENG-TR Tech. Rep, vol. 5, p. 2007, 2007.

6. J. Betthauser, D. Benavides, J. Schornick, N. O'Hara, J. Patel, J. Cole, and E. Lobaton, "Wolfbot: A distributed mobile sensing platform for research and education," in American Society for Engineering Education (ASEE Zone 1), 2014 Zone 1 Conference of the. IEEE, 2014, pp. 1-8.

7. Q. Fu, S. Yue, and C. Hu, "Bio-inspired collision detector with enhanced selectivity for ground robotic vision system," Trans. Neural Netw, vol. 17, no. 3, pp. 705-716, 2016.

8. F. C. Rind and P. J. Simmons, "Orthopteran demd neuron: a reevaluation of responses to moving objects. i. selective responses to approaching objects," Journal of Neurophysiology, vol. 68, no. 5, pp. 1654-66, 1992.

9. N. Hatsopoulos, F. Gabbiani, and G. Laurent, "Elementary computation of object approach by a wide-field visual neuron," Science, vol. 270, no. 5238, p. 1000, 1995.

10. H. Y. Meng, K. Appiah, S. G. Yue, A. Hunter, M. Hobden, N. Priestley, P. Hobden, and C. Pettit, "A modified model for the lobula giant movement detector and its fpga implementation," Computer Vision and Image Understanding, vol. 114, no. 11, pp. 1238-1247, 2010.

11. S. Yue and F. C. Rind, "Collision detection in complex dynamic scenes using an lgmd-based visual neural network with feature enhancement," IEEE Transactions on Neural Networks, vol. 17, no. 3, pp. 705-716, 2006.

12. S. B. i Badia, U. Bernardet, and P. F. Verschure, "Non-linear neuronal responses as an emergent property of afferent networks: a case study of the locust lobula giant movement detector," PLoS computational biology, vol. 6, no. 3, p. e1000701, 2010.

13. F. Arvin, J. Murray, C. Zhang, and S. Yue, "Colias: An autonomous micro robot for swarm robotic applications," International Journal of Advanced Robotic Systems, vol. 11, p. $1,2014$.

14. C. Hu, F. Arvin, and S. Yue, "Development of a bio-inspired vision system for mobile micro-robots," in Development and Learning and Epigenetic Robotics (ICDL-Epirob), 2014 Joint IEEE International Conferences on. IEEE, 2014, pp. $81-86$.

15. Q. Fu and S. Yue, "Modelling lgmd2 visual neuron system," in Machine Learning for Signal Processing (MLSP), 2015 IEEE 25th International Workshop on. IEEE, 2015, pp. $1-6$.

16. Q. Fu, C. Hu, T. Liu, and S. Yue, "Collision selective lgmds neuron models research benefits from a vision-based autonomous micro robot," in 2017 IEEE/RSJ International Conference on Intelligent Robots and Systems (IROS), Sept 2017, pp. 3996-4002.

17. C. Hu, F. Arvin, C. Xiong, and S. Yue, "Bio-inspired embedded vision system for autonomous micro-robots: The lgmd case," IEEE Transactions on Cognitive and Developmental Systems, vol. 9, no. 3, pp. 241-254, Sept 2017.

18. S. Yue and Q. Fu, "Modeling direction selective visual neural network with on and off pathways for extracting motion cues from cluttered background," in 2017 International Joint Conference on Neural Networks (IJCNN), May 2017, pp. 831838.

19. F. C. Rind, S. Wernitznig, P. Pölt, A. Zankel, D. Gütl, J. Sztarker, and G. Leitinger, "Two identified looming detectors in the locust: ubiquitous lateral connections among their inputs contribute to selective responses to looming objects," Scientific reports, vol. 6,2016 . 